\title{
一种原始真核细胞一一寇氏隐甲藻细胞中 存在类角蛋白中间纤维的证实
}

李秀芬 蔡树涛 翟中和

(北京大学生物学系, 北京 100871)

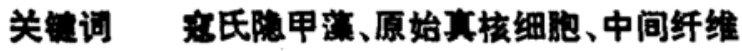

中间纤维 (intermediate filaments, IFs) 是广泛存在于高等动物细胞中的一类胞质骨架. 体系, 到目前为止, 对脊椎动物细胞中间纤维的结构、成分已经有了较为深人的了解 ${ }^{[1]}$. 进人 80 年代, 一些作者陆续报道在低等动物细胞中亦存在有中间纤维结构或其蛋白成分 ${ }^{[2,3]}$, 最近 植物细胞中间纤维的研究也取得了重要进展 ${ }^{[4-6]}$, 我们实脸室发现植物细胞中存在与动物细胞 相类似的中间纤维网络, 其蛋白成分亦具有相似的抗原性, 且能在体外重新组装成为直径约 $10 \mathrm{~nm}$ 的纤维结构. 以上研究结果说明中间纤维的存在不仅仅局限于高等动物细胞, 其起源可 能比原来所认识的更早. 为此我们选用甲藻——种原始真核生物, 代表了原核生物与真核 生物之间的过渡类型作为实验材料, 从形态学观察、蛋白成分分析和核酸的分子杂交等方面 证明了寇氏隐甲藻细胞中存在类角蛋白中间纤维体系. 这一结果为探讨中间纤维起源与进化 的问题提供了重要实验依据.

\section{一、材料与方法}

1. 细胞及其培养 寇氏隐甲藻 (Crypthecodinium cohnii) 由美国 Texas A \& M 大学 Rizzo 博士赠送, 中国科学院昆明动物研究所李靖炎教授转送, 以 E6 培养基于 $25^{\circ} \mathrm{C}$ 无 菌培养,

2. 细胞成分的选择性抽提 经 $1000 \times g$ 离心 $5 \mathrm{~min}$ 收集悬浮培养的细胞, 以含 $1 \%$ Triton X-100 的 CSK 液 (10mmol/LPIPES, $\mathrm{pH} 6.8 ; 100 \mathrm{mmol} / \mathrm{L} \mathrm{KCl} ; 300 \mathrm{mmol} / \mathrm{L}$ 蔗 糖, $3 \mathrm{mmol} / \mathrm{L} \mathrm{MgCl}_{2} ; 1 \mathrm{mmol} / \mathrm{L} \mathrm{EGTA} ; 1.2 \mathrm{mmol} / \mathrm{L} \mathrm{PMSF}$ ) 于室温处理 $10 \mathrm{~min}$, 溶去膜系 统, 可溶性蛋白随之流失; 经 $1000 \times \mathrm{g}$ 离心 $5 \mathrm{~min}$ 的沉淀物用 $\mathrm{RSB}-\mathrm{majik}$ 液 $(42.5 \mathrm{mmol} / \mathrm{L}$ Tris- $\mathrm{HCl}, \mathrm{pH} 8.3 ; 8.5 \mathrm{mmol} / \mathrm{L} \mathrm{NaCl} ; 1.2 \mathrm{mmol} / \mathrm{L}$ PMSF 和 $1 \%$ Tween-40、0.5\% 脱氧胆 酸钠) 在 $0^{\circ} \mathrm{C}$ 处理 $10 \mathrm{~min}$, 微管、微丝与其它结构蛋白被溶去; 以 $1000 \mathrm{r} / \mathrm{min}$ 被离心的沉淀物 经 DNase $\mathrm{I}(100 \mu \mathrm{g} / \mathrm{ml})$ 于 $25^{\circ} \mathrm{C}$ 处理 $30 \mathrm{~min}$, 然后加 $1 \mathrm{~mol} / \mathrm{L}$ 硫酸胺至终浓度为 $0.25 \mathrm{~mol} /$ L, DNA 与组蛋白被除去, 取得的沉淀物主要为细胞的核骨架与中间纤维结构.

3. DGD 包埋-去包埋电镜样品制备 经上述分级抽提的样品先用 $0.25 \%$ 戊二醛固定 $30 \mathrm{~min}$, 再用 $0.1 \% \mathrm{OsO}_{4}$ 固定 $1 \mathrm{~h}$, 经系列乙醇脱水, 正丁醇过渡, 用 $\mathrm{DGD}$ 浸透包埋, 超薄切 片以正丁醇溶去包埋剂, 漫人乙醇, 经乙酸异戊酯置换后于二氧化碳临界点千燥. 不经染色.

1992-02-29 收稿, 1992-08-22 收修改稿 
JEM $100 C X$ 电镜观察.

4. 免度印迹反应上述分级抽提的样品经 SDS 聚丙烯酰胺凝胶电泳, 再转移至硝酸纤 维素滤膜上, 用 3\%牛血清蛋白封闭后, 与动物角蛋白单克隆抗体 AE3 (纽约大学孙同天教 授赠, 广谱识别动物碱性角蛋白)于 $37^{\circ} \mathrm{C}$ 反应 $1 \mathrm{~h}$, 再与碱性磷酸酶标记的第二抗体于: $37^{\circ} \mathrm{C}$ 反 应 $1 \mathrm{~h}$, 经洗涤后以氯化硝基四氮唑蓝、5-溴-4-氯-3-吲哚-磷酸甲苯胺蓝显色.

5. 间接免度荧光染色 经上述系列抽提的细胞以冷乙醇: 丙酮 (1:1) 固定 $10 \mathrm{~min}$, 经 PBS 洗涤后与 AE3 抗体反应, 于 $37^{\circ} \mathrm{C}$ 作用 $1 \mathrm{~h}$, 经 PBS 洗涤后再与 FITC 苂光素标记的 第二抗体于 $37^{\circ} \mathrm{C}$ 作用 $1 \mathrm{~h}$, 用 PBS 洗涤后加甘油封片.

6. 中间纤维蛋白的体外亚组经上述抽提得到的蛋白样品溶于样品溶解液中（8mol/ $\mathrm{L}$ 㽷素, $10 \mathrm{mmol} / \mathrm{L}$ Tris-HCl, pH7.5, $50 \mathrm{mmol} / \mathrm{L}$ 疏基乙醇), 于 $25^{\circ} \mathrm{C}$ 过夜, 以 $200000 \times \mathrm{g}$ 在 $20^{\circ} \mathrm{C}$ 离心 $2 \mathrm{~h}$, 弃去沉淀, 用 $2000 \times$ 体积透析液 $(10 \mathrm{mmol} / \mathrm{L}$ Tris $-\mathrm{HCl}, \mathrm{pH} 7.5 ; 25 \mathrm{mmol} /$ L 琉基乙醇; $1.2 \mathrm{mmol} / \mathrm{L}$ PMSF) 于 $4^{\circ} \mathrm{C}$ 透析过夜; 可以观察到浊度增大, 然后取材悬滴制 样, 以 $0.5 \%$ 醋酸铀染色 $30 \mathrm{~s}$, JEM $100 \mathrm{CX}$ 电镜观察.

7. Southern 杂交甲藻细胞经液氮研磨, 酚、氯仿除去蛋白, 再用乙醇沉淀得到总 DNA.

取 $10 \mu \mathrm{g}$ 甲藻总 DNA 用 Hind III 酶在 $37^{\circ} \mathrm{C}$ 消化 $2 \mathrm{~h}$ 后进行琼脂糖凝胶电泳, 电泳凝胶 经变性、中和与平衡处理后, 用吸水纸转移至硝酸纤维滤膜上. 滤膜在 $60^{\circ} \mathrm{C}$ 经 $8 \mathrm{~h}$ 烤干, 与预 杂交液 ( $50 \%$ 甲酰胺, $5 \times$ SSPE, $5 \times$ Denhardt, $0.1 \%$ SDS, $0.5 \%$ 脱脂奶粉) 手 $42^{\circ} \mathrm{C}$ 反应 $4 \mathrm{~h}$, 再加人经煮沸变性的 K3 角蛋白 cDNA 探针(纽约大学孙同天教授赠, 以 ${ }^{32} \mathrm{P}-\mathrm{dCTP}$ 标 记),于 $42^{\circ} \mathrm{C}$ 反应 $12 \mathrm{~h}$, 洗涤后封人塑料薄膜, 在一 $70^{\circ} \mathrm{C}$ 用 X 光片感光.

\section{二、结果与讨论}

1. 陆氏隐甲藻细胞中存在类中间纤维网络 甲藻细胞经选择性系列抽提后的样品用 DGD 包埋一去包埋剂的超薄切片经二氧化碳临界点干燥后, 在透射电镜下可以观察到甲藻细 胞质中存在一致密精细的纤维网络结构 (图 1), 其单丝的直径约在 $10 \mathrm{~nm}$ 左右. 它们同动物 细胞中间纤维一样, 对非离子去垢剂和高盐溶液的处理有较高的稳定性. 说明甲藻细胞质中 具有与哺乳动物中间纤维相似的中间纤维结构.

2. 甲落细胞的类中间纤维含有类角蛋白成分 上述选择性抽提得到的纤维蛋白组分经 SDS 聚丙烯酰胺凝胶电泳分析, 其蛋白条带较为复杂 (图 2b), 可能与甲藻核骨架组分复杂有 关 ${ }^{\mathrm{B} 1}$. 用从动物细胞中得到的碱性角蛋白单抗 AE3 进行免疫印迹反应, 发现其中一个蛋白条 带与之呈明显的阳性反应, 其分子量约为 $67 \mathrm{kD}$ (图 $2 \mathrm{c}$ ). 用上述哺乳动物角蛋白单抗对甲藻 细胞进行免疫荧光染色, 细胞质内出现较强的阳性反应, 荻光呈弥散分布 (图 3), 而核区部分 则是阴性反应. 免疫苂光染色的结果为形态学与免疫检测的实验结果提供了佐证, 表明在甲 藻细胞中不仅存在类中间纤维的形态结构, 而且其成分包含了与动物细胞相似的类角蛋白成 分.

3. 甲落的类中间纤维蛋白成分能在体外重组䒾成 $10 \mathrm{~nm}$ 纤维 能在体外自主装配是中 间纤维蛋白的一个主要特征 ${ }^{[9]}$. 经上述抽提得到的核骨架中间纤维蛋白组分经变性剂处理, 此时纤维蛋白以亚基的形式存在,经透析除去变性剂后, 取样悬滴进行负染色, 电镜下观察到 抽提的蛋白组分在体外重新组装成直径约 $10 \mathrm{~nm}$ 的均匀长丝结构 (图 4), 具有典型的中闹纤 


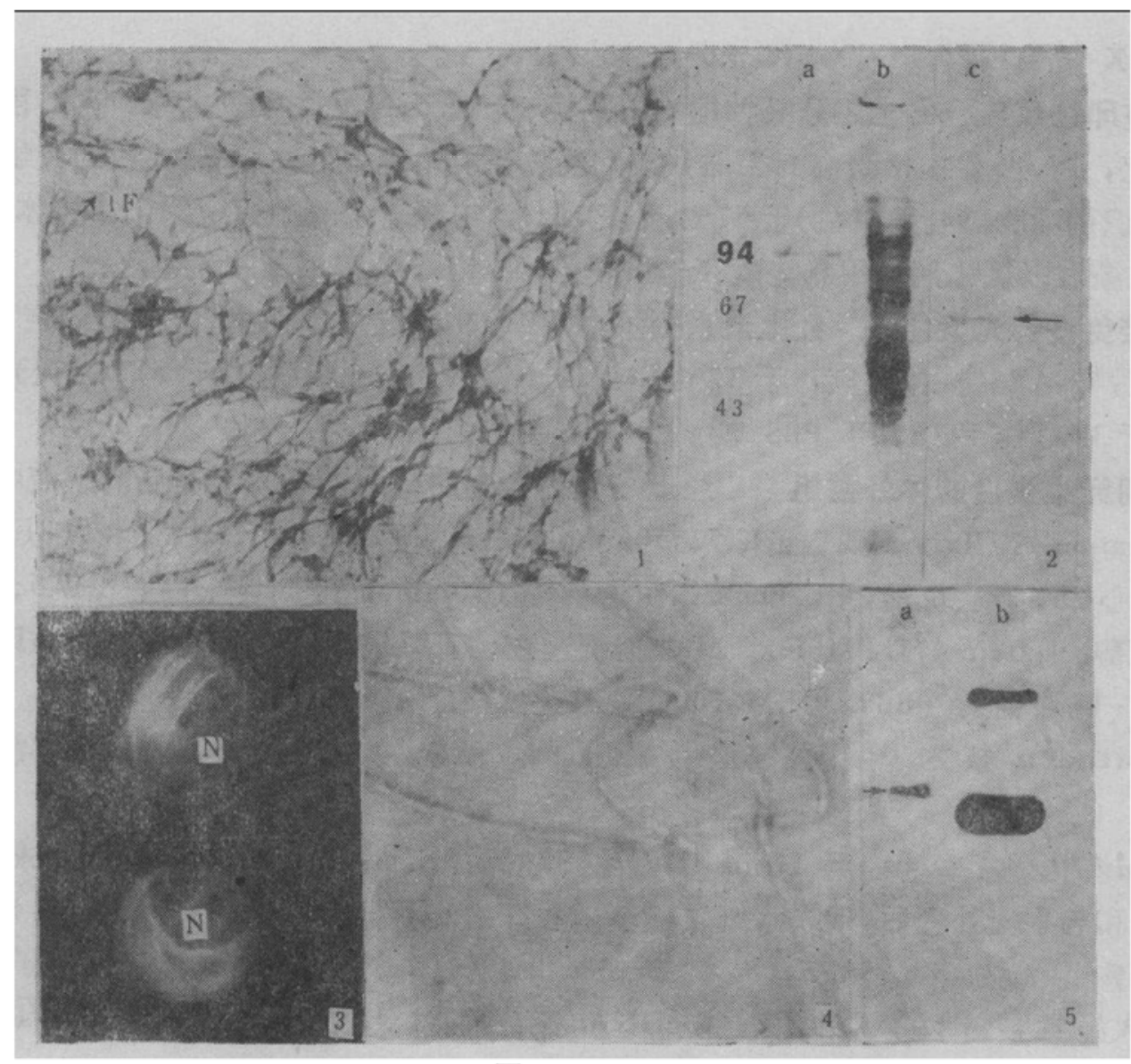

图 1-5

1. DGD 超薄切片显示经选择性抽提后, 甲藻细胞胞质内可见类中间纤维构成的精细网络,其单丝直经 约 $10 \mathrm{~nm}(\times 24000)$. 2.危疫印迹法显示中间纤维中的类角蛋白成分 a. 标准蛋白,示分子盗大小,单 位 $\mathrm{kD}$; b. SDS-PAGE 分析经系列抽提得到的纤维蛋白成分; c. 免度印迹分析显示与角蛋白单抗 呈阳性反应的条带 $(\rightarrow)$, 其分子量约为 $67 \mathrm{kD}$. 3. 间接免度苂光染色, 显示阳性反应出现于胸质区域, $N$ 为柆区 $(\times 800)$ ，4.负染法显示体外重新组装的类中间纤维结构,其直径约 $10 \mathrm{~nm}(\times 16000)$. 5. Southern 杂交显示甲基因组含有与动物细胞角蛋白基因具有同源性的核酸片段. 3. 甲 藻基因组 DNA 与动物角蛋白 cDNA 进行分子杂交结果, 显示阳性条带 $(\rightarrow)$, 其分子量约为 $3.5 \mathrm{~kb} ;$ b. 含有 K3 cDNA 的质柆, 作为 Southern 杂交的阴性对服

维的形态. 至此我们可以明确地说明, 甲藻细胞内的中间纤维蛋白与哺乳动物细胞和植物细 胞一样, 具有体外重组装的能力.

4. 甲落细胞基因组中含有唃乳动物角蛋白基因的同源序列 以哺乳动物角蛋白的 cDNA 为探针与甲藻细胞基因组 DNA 进行 Southern 杂交, 结果显示有一明显阳性反应条 带 (图 5), 限制酶切片段大小约为 $3.5 \mathrm{~kb}$, 即甲藻基因组中含有与哺乳动物编码角蛋白的基因 具有同源性的核酸片段。这一同源序列的发现,为进一步的工作提供了良好基础.

根据以上形态结构的观察、蛋白成分的分析、中间纤维蛋白自组装的特征与 DNA 同源序 列的分析, 我们首次证实在现存原始的真核生物寇氏隐甲藻细胞中已经出现了类中间纤维体 系,这一发现为解决生物进化过程中中间纤维的起源问题提供了重要依据.

致谢本文所涉及免疫菼光染色技术与范檘同学合作完成, 特此表示谢意.

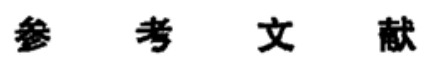

[1] Steinert, P. M., Cell, 42(1985), 414, 419. 
[2] Numata, O., Watanabe, Y., J. Biochem., 90(1982), 1563-1573.

[3] Koury, S. T., Edeart, B. S., J. Cell Biol., 97(1983), 223a.

[4] Su, F. et al., Cell Research, 1(1990), 9-13.

[5] 邢力等,科学通报, 36(1991), 8: 623-626.

[6] Yang, C. et al., Proceedings of Int. 4th Beijing Conference on Instrumental Analysis, Beijing, 1991, A133- 134.

[7] Dodge, J. D., Int. Cong. Ser. Excerpsa Med., 91(1965), 264-265.

[8] 蔡树涛等,实验生物学报,24(1991),261-271.

[9] Steinert, P. M., Idler, W. W., J. Mol. Biol., 108(1976), 547-567. 